\title{
Significance of high resolution MRI in the identification of carotid plaque
}

\author{
YONG SUN* ${ }^{*}$ LEI XU*, YAN JIANG, MING MA, XIN-YI WANG and YING XING \\ Department of Neurology, China-Japan Union Hospital of Jilin University, Changchun, Jilin 130033, P.R. China
}

Received February 4, 2020; Accepted July 1, 2020

DOI: $10.3892 /$ etm.2020.9091

\begin{abstract}
The stability of carotid artery plaque serves a key role in the occurrence of stroke. The present study was based on the recruitment of patients with acute ischemic cerebrovascular disease. High-resolution magnetic resonance imaging (HR-MRI) was used to identify the nature of carotid artery plaque, and the results were then used to manage the high-risk group of stroke. The patients were divided equally into a symptomatic group (36 cases) and an asymptomatic group (36 cases). According to the degree of carotid artery stenosis, the patients were divided into mild, moderate and severe stenosis groups, each group comprising 12 patients, and HR-MRI was performed. The proportion of patients with vulnerable plaque in the symptomatic group was higher compared with that in the asymptomatic group $(\mathrm{P}<0.05)$. The more severe the stenosis, the higher the proportion of vulnerable plaque that was identified $(\mathrm{P}<0.05)$. Compared with carotid ultrasound, HR-MRI was indicated to have the capability to both identify and quantify the different components in the plaque, allowing an assessment of its properties. In conclusion, the present study demonstrated that carotid HR-MRI is able to distinguish and quantify the different components of plaque, which may prove to be helpful for the hierarchical management of a population at high risk of stroke.
\end{abstract}

\section{Introduction}

The incidence of stroke is increasing on an annual basis worldwide, with high disability and mortality rates (1). In excess of two-thirds of stroke cases are of ischemic stroke, which has become the second leading cause of mortality and disability worldwide (2). Stenosis that is caused by the formation and

Correspondence to: Dr Ying Xing, Department of Neurology, China-Japan Union Hospital of Jilin University, 126 Xiantai Street, Changchun, Jilin 130033, P.R. China

E-mail: xingying1970@163.com

*Contributed equally

Key words: carotid artery, high-resolution MRI, carotid ultrasound, vulnerable plaque, stenosis development of atherosclerosis is one of the main risk factors of cerebrovascular disease, especially ischemic cerebrovascular disease, which accounts for $19-35 \%$ of all cases (3), and is closely associated with high mortality rate due to acute cerebral infarction $(4,5)$. Therefore, the management of ischemic stroke to achieve the risk stratification of patients is becoming increasingly important, where not only the severity of carotid stenosis is evaluated, but also the stability of carotid plaque. Previous studies have indicated that the components of atherosclerotic plaque will also affect its stability, leading to the emergence of vulnerable plaque $(6,7)$. The presence of components of vulnerable plaque provide an indication that the probability of plaque rupture is high, with plaque rupture subsequently leading to thrombus events (8). In a large-scale study of carotid plaque histology, it was demonstrated that the histological characteristics of carotid plaque instability are closely associated with the risk of stroke (9), and the presence and composition of vulnerable plaque are also associated with the occurrence of ipsilateral cerebrovascular events (10). Therefore, vulnerable plaque formation fulfills an important role in the prevention and control of cerebrovascular diseases.

With the development of imaging technology, it has been previously demonstrated that high-resolution magnetic resonance imaging (HR-MRI) exhibits high sensitivity and specificity in terms of the detection of carotid vulnerable plaque (11). Collected data has indicated that the sensitivity and specificity of this technique can be as high as $90-100 \%(12,13)$. HR-MRI is able to clearly discern the structural characteristics of the vascular wall, the shape and size of the arterial plaque and its specific components. However currently, relatively few studies have been published on this topic, and those on carotid plaque models with different degrees of stenosis are scarce. Therefore, in the current study, the clinical data of corresponding patients was collected for analysis to explore the value of HR-MRI in the identification of vulnerable carotid plaque.

\section{Materials and methods}

Patients. Between March 2018 and March 2019, 72 patients in the Department of Neurology China-Japan Union Hospital of Jilin University were selected, including 49 males and 23 females, aged between 45 and 82 years. The present study was approved by the Ethics Committee of China-Japan Union hospital of Jilin University (approval no. 2020010812; 
Changchun, China). Patients who participated in this research had complete clinical data. Signed informed consents were obtained from the patients and/or the guardians.

Inclusion and exclusion criteria. Patients were included in the present study if they met the following criteria: i) Carotid plaque and different degrees of carotid stenosis had been identified by carotid ultrasound; ii) the diagnosis of acute cerebrovascular disease conformed to the Chinese guidelines for the diagnosis and treatment of acute ischemic stroke (2018 edition) (14); iii) the age of the patient fell within the range 18-85 years; and iv) the patients and their families were willing to sign informed consent forms.

The exclusion criteria were as follows: i) The patient also suffered from serious heart disease and arrhythmia (such as atrial fibrillation); ii) there were foreign bodies in the body that could not be examined by MRI, such as metal prostheses or pacemakers; iii) the patient was convulsing, and unable to cooperate with the examination; iv) the patients had a severe high fever; v) the patient experienced claustrophobia, drug or alcohol abuse, or other mental disorders; and vi) the MRI quality score was $\leq 2$ (as defined by the presence of motion and blood flow artifacts in the image, such that the plaque components could not be clearly identified).

Study groups. Patients who were admitted to the hospital between March 2018 and March 2019 were divided into a symptomatic group (group A) and an asymptomatic group (group B), according to whether the patient was suffering from acute ischemic cerebrovascular disease. After the degree of stenosis had been assessed by carotid ultrasound, patients were further divided into mild stenosis (stenosis degree $<50 \%$; groups $\mathrm{A} 1$ and $\mathrm{B} 1$ ), moderate stenosis (stenosis degree 50-69\%; groups A2 and B2) and severe stenosis (stenosis degree $\geq 70 \%$; groups A3 and B3) groups. A total of 12 patients were included in each group, and a total of 72 patients were included in the study.

\section{Collection of image data}

Cervical vascular examination. The carotid ultrasound system utilizes a domestic Mindray DC-8 color Doppler ultrasound imaging instrument (Mindray Medical International Limited) with a probe frequency of $9 \mathrm{~Hz}$. The bilateral carotid arteries were examined separately, and the morphology of carotid plaque was observed according to color Doppler flow imaging. The echo characteristics were subsequently analyzed. The plaque size, and peak and diastolic peaks were measured at their narrowest dimensions, and the sizes of the distal segments of the lumen were also measured. All carotid ultrasound procedures were performed by an experienced sonographer.

Carotid HR-MRI examination. In order to perform the carotid HR-MRI examinations, the Siemens Skyra 3.0T nuclear magnetic resonance scanning system (Siemens Healthineers) was employed. The coil used was the Siemens head-and-neck combined coil. The patient was instructed to lay down on the examination bed, where the head was later tilted. Prior to the scan, the examinee was subjected to a longer scan time, where the examinees were instructed to allow their body to relax. After having adjusted themselves to a comfortable position, the patients were restricting from moving their bodies freely during the scanning process in order to minimize swallowing, to reduce artifacts during imaging and to improve image quality. In terms of HR-MRI, carotid plaque three-dimensional time-of-flight (3D-TOF) scans [time of repetition (TR), $29 \mathrm{msec}$; time of echo (TE), $4 \mathrm{msec}$; field of view (FOV), 160/180 mesc], T1W1 black blood scans (TR, $800 \mathrm{msec}$; TE, $10 \mathrm{msec}$; FOV, 160/180 msec) and T2WI black blood nuclear scans (TR, $3500 \mathrm{msec}$; TE, $60 \mathrm{msec}$; FOV, $160 / 180 \mathrm{msec}$ ) were sequentially performed on each patient. Concerning the neck positioning image scanning method, the following procedure was followed: The sagittal position was centered at the position of the 3-4 level of the flat cervical vertebra, and in the coronal and transverse positioning images, the target area was placed in the center of the field-of-view (FOV) frame.

Image post-processing. The ultrasound reports were issued after evaluation and analysis of the results obtained from the cervical vascular ultrasound. These reports were produced by an ultrasound doctor with a deputy chief physician, or by a physician with a higher status. A multi-contrast MRI sequence image obtained after HR-MRI by an experienced radiologist combined with a Magnetic resonance-Vulnerable plaque diagnosis technology (MR-VPD) examination was processed using the MRI-PlaqueView ${ }^{\mathrm{TM}}$ software (V2.1) (V2.1; VPDiagnostics) to analyse and report on the data. The evaluation included a determination of the degree of vascular stenosis according to the following equation: Degree of vascular stenosis $=(\mathrm{B}-\mathrm{A}) / \mathrm{B} \times 100 \%$, where $\mathrm{A}$ is the narrowest diameter of the stenosis, and $B$ is the normal diameter of the stenosis telecentric end. Diagnostic criteria of carotid stenosis were then consulted: i) $<50 \%$, mild stenosis; $>70 \%$, severe stenosis (15).

The acoustic characteristics of the carotid ultrasound were subsequently analyzed in order to provide the accurate classification of stenosis, and to classify stenosis into mild, moderate or severe stenosis, according to the standard protocol (14). Before analyzing the images using the MRI-PlaqueView ${ }^{\mathrm{TM}}$ software, the HR-MRI multi-contrast images of the neck were evaluated for image quality. According to the evaluation criteria (16), a quality score between 1 (for low quality) and 4 (for high quality) was assigned. The American Heart Association (AHA) plaque typing method was followed, which was based on the modified plaque classification standard for nuclear magnetics (17) in which plaque types I-II, III, VII and VIII are classified as stable plaque, and plaque types IV-V and VI are categorized as vulnerable plaque. Specifically, the descriptions of the plaque types are as follows: Type I-II, near-normal wall thickening without calcification; type III, diffuse thickening or small eccentric non-calcified plaque under the endometrium; type IV-V, a lipid or necrotic core exists in the plaque, which is surrounded by fibrous tissue and may have calcification; type VI, complex plaque, which may have surface defects, bleeding, or thrombosis; type VII, calcified plaques; and type VIII, fibrous plaque without a lipid core that may have small calcifications.

Statistical analysis. The obtained research data were statistically analyzed using SPSS software version 19.0 (IBM Corp.), 
and comparisons between the data groups were performed by using the $\chi^{2}$ test. $\mathrm{P}<0.05$ was considered to indicate a statistically significant difference.

\section{Results}

Comparison of the number of cases with vulnerable plaque in the symptomatic and asymptomatic groups. The number of cases with vulnerable plaque in groups A and group B were statistically analyzed. A total of 27 cases with vulnerable plaque were identified in group A $(27 / 36 ; 75.0 \%)$, and the number of cases with vulnerable plaque in group B was indicated to be 17 cases $(17 / 36 ; 47.2 \%)$; therefore, the proportion of patients with vulnerable plaque in group A was greater compared with that in group B. The number of unstable plaques in group A was significantly higher compared with that in group $\mathrm{B}(\mathrm{P}<0.05$; Fig. 1).

Comparison of the number of vulnerable plaque in different stenosis groups. The number of cases containing vulnerable plaque in the $\mathrm{A} 1+\mathrm{B} 1, \mathrm{~A} 2+\mathrm{B} 2$ and $\mathrm{A} 3+\mathrm{B} 3$ groups were counted, and the number of cases containing vulnerable plaque in the $\mathrm{A} 1+\mathrm{B} 1$ group was indicated to be 8 cases $(8 / 24 ; 33.3 \%)$. The number of cases containing vulnerable plaque in the $\mathrm{A} 2+\mathrm{B} 2$ group was $15(15 / 24 ; 62.5 \%)$, whereas the number of cases with vulnerable plaque in the A3+B3 group was 21 (21/24; 87.5\%). The proportions of vulnerable plaque in the three groups with different degrees of stenosis were therefore significantly different $(\mathrm{P}<0.05)$, and a comparison of the groups revealed that, the more severe the stenosis, the higher the proportion of vulnerable plaque identified ( $\mathrm{P}<0.05$; Fig. 2$)$.

Carotid ultrasound and HR-MRI plaque properties and image comparison

Comparison of plaque properties in carotid ultrasound and $H R-M R I$ in each group. In the mild stenosis group $(\mathrm{n}=24)$, a total of 5 hypoechoic plaques, 17 hypoechoic and mixed echo plaques, 6 equal echo plaques and 1 strong echo plaque were screened using carotid ultrasound. A total of 6 type I-II plaques, 14 type III plaques, 5 IV-V plaques, 3 type VI plaques, and 1 type VII plaque were detected by HR-MRI. In the moderate stenosis group $(\mathrm{n}=24), 9$ hypoechoic plaques, 20 hypoechoic and mixed echo plaques, 2 equal echo plaques and 1 strong echo plaque were screened by carotid ultrasound. A total of 13 type III plaques, $8 \mathrm{IV}-\mathrm{V}$ plaques, 10 type VI plaques, and 1 VII type plaque were detected using HR-MRI. Finally, in the severe stenosis group $(n=24), 13$ hypoechoic plaques, 18 hypoechoic and mixed echo plaques, 3 equal echo plaques and 1 strong echo plaque were screened using carotid ultrasound. And in the severe stenosis group, 2 type III plaques, 5 IV-V plaques, 24 type VI plaques, 2 type VII plaques and 2 type VIII plaques were detected using HR-MRI (Figs. 3 and 4).

Comparison of image characteristics of each group in carotid ultrasound and HR-MRI. Carotid ultrasound and HR-MRI were consistent in the assessment of plaque vulnerability for the three established groups: mild, moderate and severe stenosis. Carotid artery ultrasound revealed low echo and mixed echo plaques, multiple hypoechoic and mixed echo

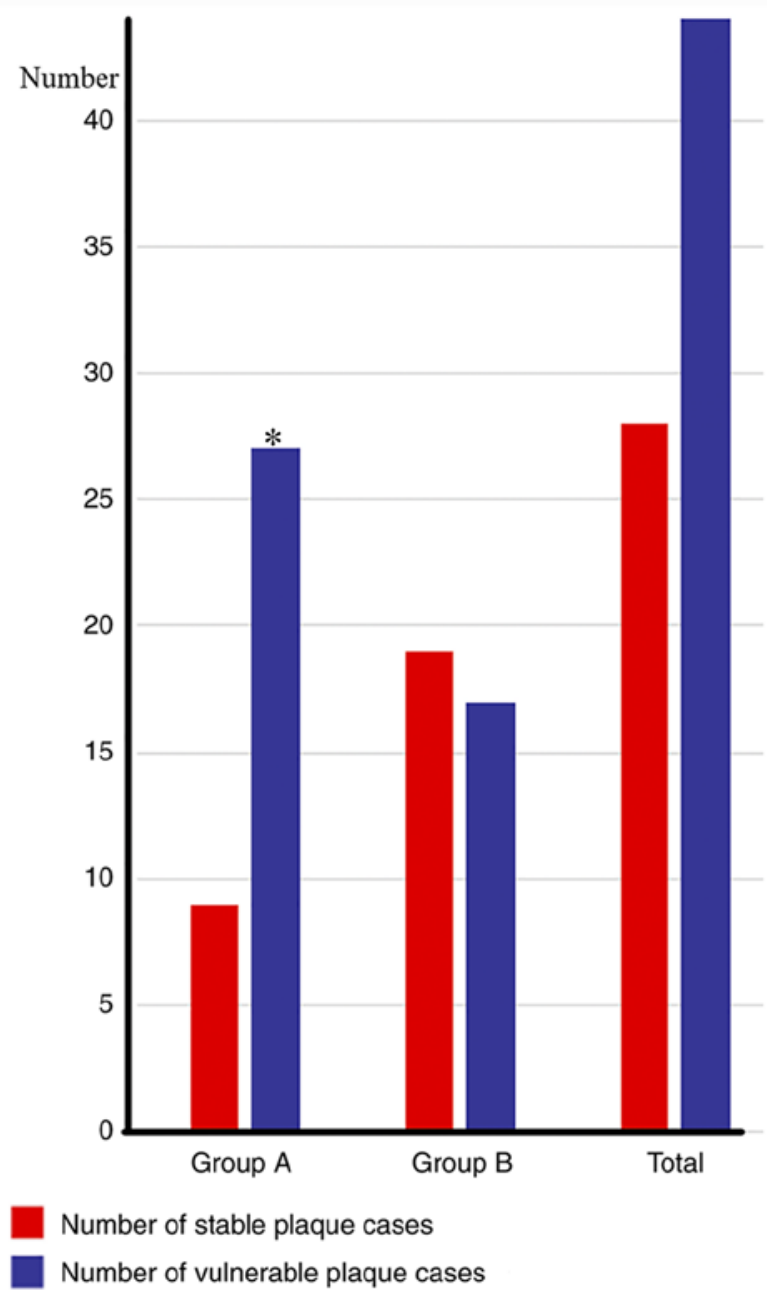

Figure 1. Comparison of the number of cases with vulnerable plaque in symptom group and non-symptomatic group. ${ }^{*} \mathrm{P}<0.05$ vs. Group $\mathrm{B}$

plaques and hypoechoic and mixed echo plaques on the wall of the carotid artery (Figs. 5-7). In comparing the results, carotid ultrasound was demonstrated to have the ability to identify plaque, and the plaque position, size and shape were all displayed. The characteristics of plaque were assessed according to the plaque echo intensity. HR-MRI was able to identify different components inside the plaque. After subsequent processing with MRI-PlaqueView ${ }^{\mathrm{TM}}$ software, it was found that the size of the plaque was $1.36 \times 0.26 \mathrm{~cm}$, where the lumen of the internal carotid artery was narrow and the inner diameter of the narrower section was $0.39 \mathrm{~cm}$ (Fig. 5). The diameter stenosis rate was calculated to be $47 \%$ in the mild stenosis group (Fig. 5). By contrast, the larger plaque was revealed to be located at the bifurcation of the carotid artery, where the size was $1.43 \times 0.43 \mathrm{~cm}$ and the lumen of the internal carotid artery was narrow, with a stenosis rate of $50-69 \%$ in the moderate stenosis group (Fig. 6). In the severe stenosis group, the larger plaque on the left side is located at the bifurcation of the carotid artery, where the size of the plaque is $2.08 \times 0.63 \mathrm{~cm}$ (Fig. 7).

The MRI-PlaqueView ${ }^{\mathrm{TM}}$ software made it possible to quantify the different components inside the plaque through the multi-dimensional color display. The MRI-PlaqueView ${ }^{\mathrm{TM}}$ is more effective for the identification of vulnerable plaques, 


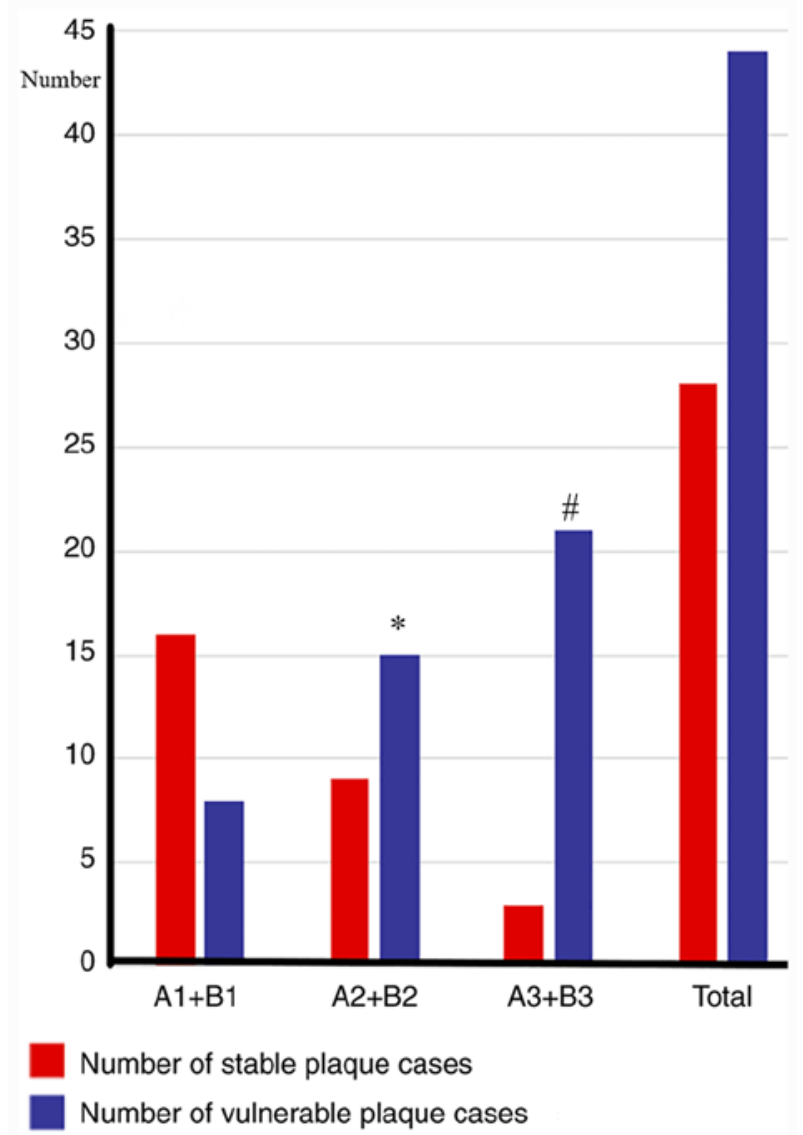

Figure 2. Comparison of cases with vulnerable plaques in different stenosis groups. " $\mathrm{P}<0.05$ vs. $\mathrm{A} 1+\mathrm{B} 1$ and ${ }^{\#} \mathrm{P}<0.05$ vs. $\mathrm{A} 2+\mathrm{B} 2$.

and there are a number of clear advantages compared with ultrasonography (Figs. 5-7).

\section{Discussion}

Atherosclerosis is an inflammatory disease involving the blood vessels of the entire body (18). Epidemiological studies have revealed that $\sim 15 \%$ of all stroke cases occur secondary to carotid atherosclerosis (19). Stenosis caused by atherosclerosis results in 10-20\% of all cases of cerebral infarction worldwide, or transient ischemic attack (20). Over the course of the last 20 years, numerous clinical trials (21-23) have used carotid stenosis as a means of risk stratification. Clinical studies have convincingly demonstrated that certain plaque components, that is, intraplaque hemorrhage and lipid core, are associated with plaque instability and plaque rupture, and also with an increased risk of stroke (24). In the present clinical study, the proportion of patients with vulnerable plaque in the symptomatic group was indicated to be higher compared with that in the asymptomatic group, a finding that was similar to a previous study (25). The results of the aforementioned study (25) revealed that the main causes of ipsilateral cerebrovascular disease were as follows: i) Ruptured vulnerable plaque is able to cause cerebral embolism with blood flow; and ii) the surface of vulnerable plaque is not smooth, thereby facilitating the process of adsorption of platelets and coagulation factors. The plaque composition has also been studied in a postmortem series, which demonstrated that intracranial plaques associated

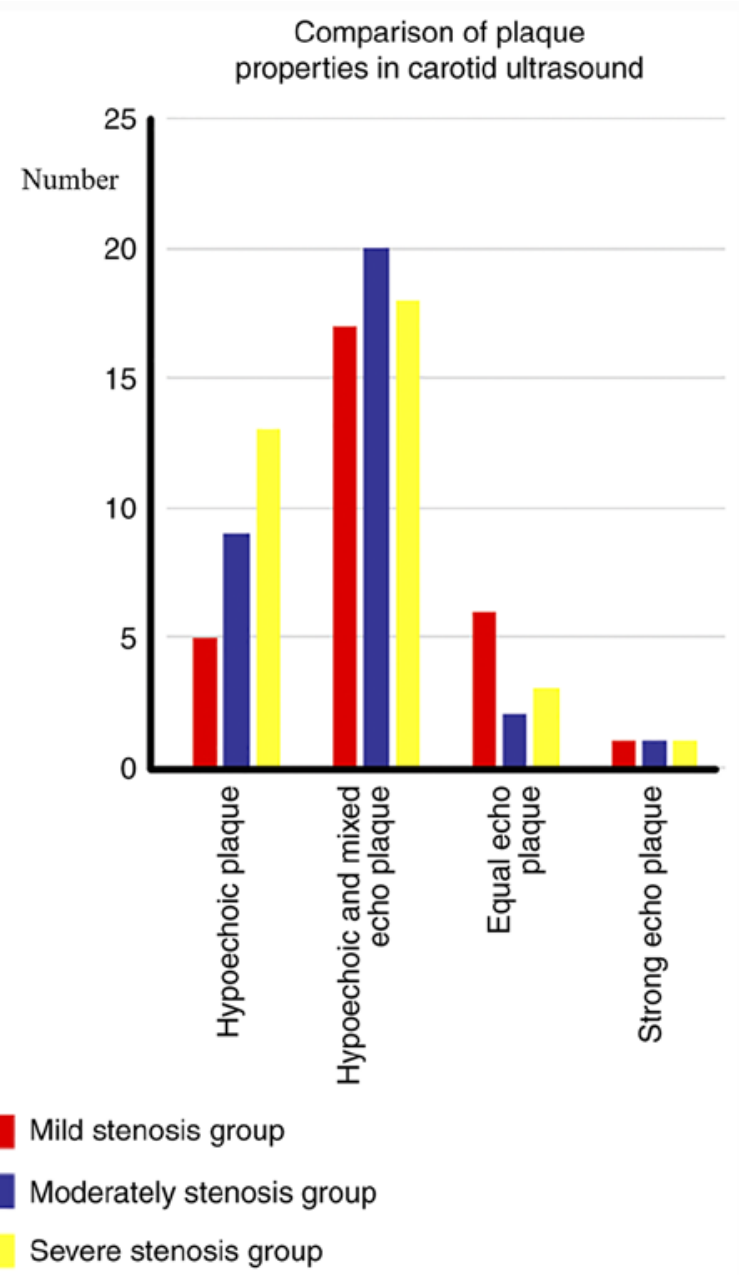

Figure 3. Comparison of plaque properties in carotid ultrasound between the mild, moderately and severe stenosis groups.

with infarct had i) a higher area of lipid core, ii) a higher frequency of neovasculature and thrombus, iii) a trend toward a higher frequency of intraplaque hemorrhage (25), meaning the more unstable the intracranial plaque, the higher the probability of cerebral infarction. This may lead to thrombosis on the plaque surface due to the coagulation pathway (26). On the one hand, thrombosis on the plaque surface can cause the continuous formation and expansion of plaque, with the blockage of blood vessels occurring in serious cases, resulting in insufficient blood perfusion; and on the other hand, it can result in the rupture of thrombus and cerebral embolism $(27,28)$. At present, relatively few studies have been published on this topic, and in assessing different degrees of stenosis according to the carotid plaque model, the nature of the plaque has been infrequently studied. Atherosclerosis provides the underlying pathological basis of vascular diseases. The plaque or stenosis resulting from atherosclerosis is the key factor for the progression and recurrence of cerebrovascular diseases (29). Only a narrow percentage difference in the extent of stenosis caused by the blockage of the lumen determines whether carotid endarterectomy and carotid stent placement will be necessary for the patient (30). Dong et al (31) found that the proportion of vulnerable plaque was also high in patients without any degree of vascular stenosis. Therefore, taking only carotid stenosis as 


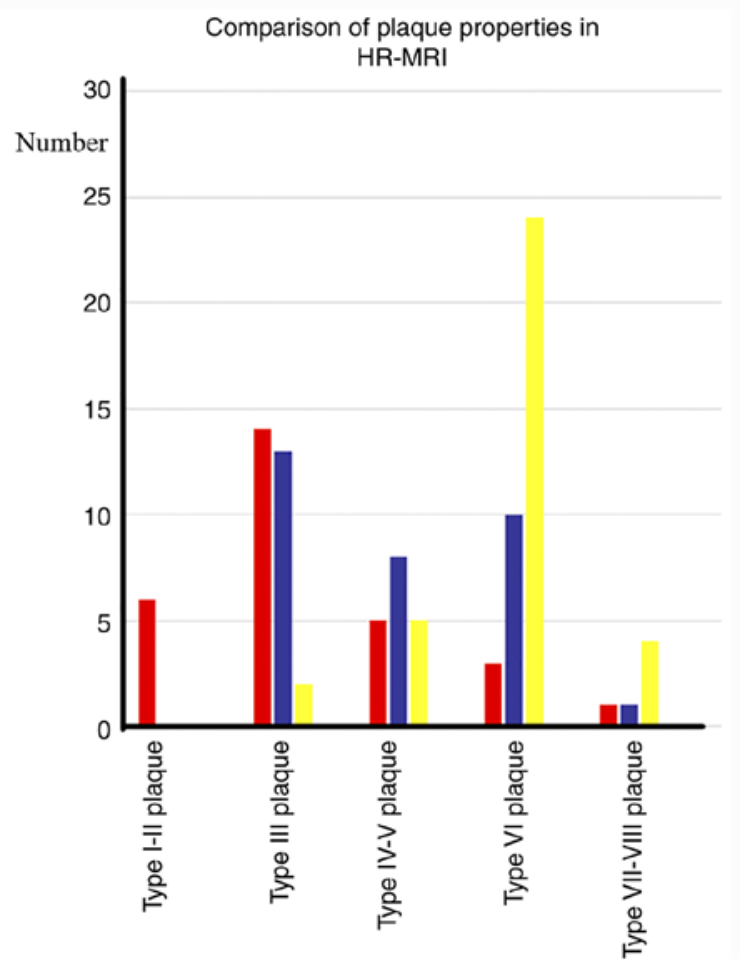

Mild stenosis group

Moderately stenosis group

Severe stenosis group

Figure 4. Comparison of plaque properties in HR-MRI in the mild, moderately and sever stenosis groups. HR-MRI, high-resolution magnetic resonance imaging.
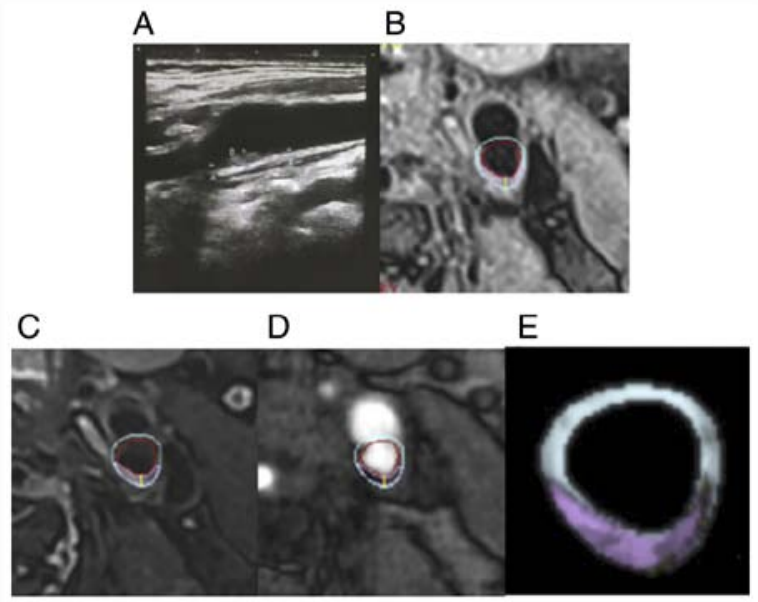

Figure 5. Comparison of carotid ultrasound and cervical HR-MRI images in the mild stenosis group. (A) Representative carotid artery ultrasound images, (B) T1, (C) T2, (D) TOF and (E) vessel examinations. Green, fiber; white, calcification; yellow, lipid-rich core; pink, loose substrate area; red, lumen or hemorrhage; blue, tube wall. HR-MRI, High resolution magnetic resonance imaging; TOF, time-of-flight.

the clinical intervention standard, the risk assessment will lead to a missed diagnosis or misdiagnosis of some patients. In addition, a previous study (32) have demonstrated that compared with vascular stenosis, vulnerable plaque is also associated with a great risk of vascular disease. With an increase in the
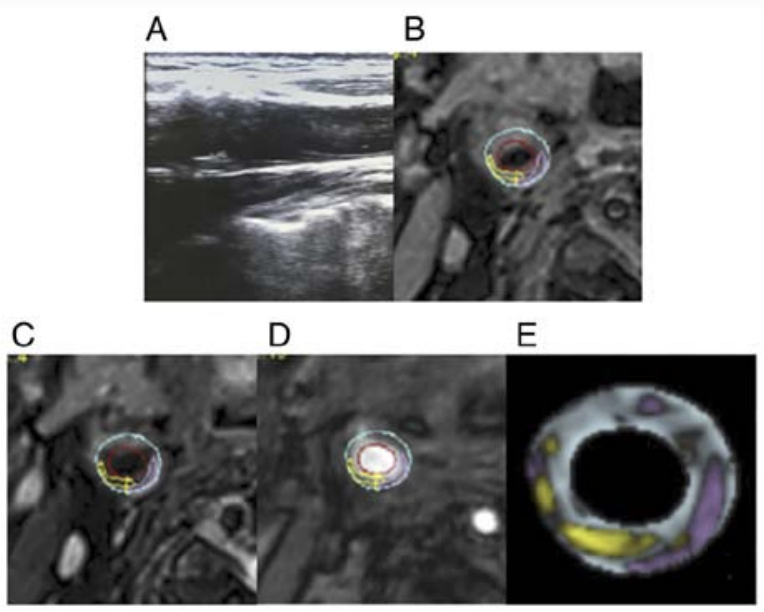

Figure 6. Comparison of carotid ultrasound and cervical HR-MRI images in the moderate stenosis group. (A) Representative carotid artery ultrasound images. (B) T1, (C) T2, (D) TOF and (E) vessel examinations. Green, fiber; white, calcification; yellow, lipid-rich core; pink, loose substrate area; red, lumen or hemorrhage; blue, tube wall. HR-MRI, high-resolution magnetic resonance imaging; TOF, time-of-flight.
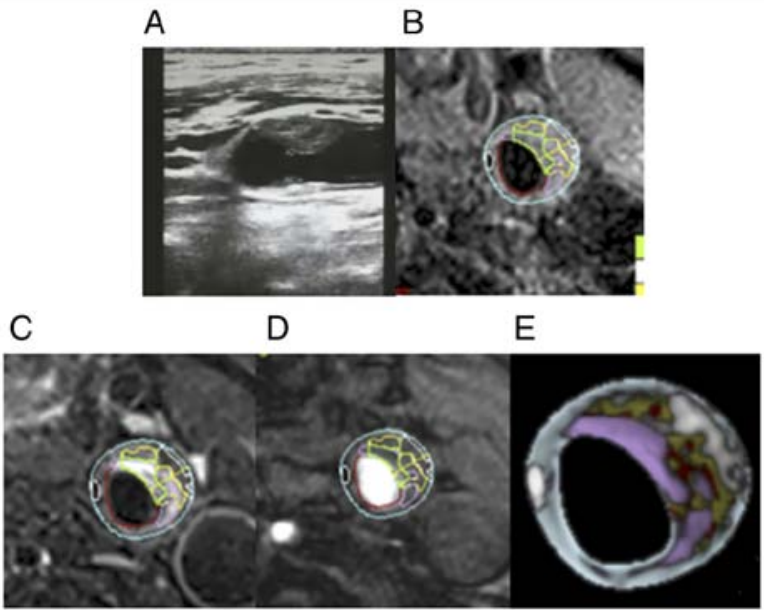

Figure 7. Comparison of carotid ultrasound and cervical HR-MRI images in the severe stenosis group. (A) Representative carotid artery ultrasound images. (B) T1, (C) T2, (D) TOF and (E) vessel examinations. Green, fiber; white, calcification; yellow, lipid-rich core; pink, loose substrate area; red, lumen or hemorrhage; blue, tube wall. HR-MRI, high-resolution magnetic resonance imaging; TOF, time-of-flight.

degree of stenosis, the prevalence of the lipid-rich necrotic core (LRNC) also increases significantly. A previous study reported that, for patients identified as having an $80-99 \%$ extent of carotid artery stenosis, $91.8 \%$ of the patients also had LRNC lesions (33). In patients with mild stenosis of carotid artery, the proportion of intraplaque hemorrhage (IPH) was indicated to be $7 \%$ (34). Therefore, attention must be paid not only to the degree of stenosis, but also to the stability of plaque associated with the different degrees of stenosis. In the present clinical study, it was demonstrated that the proportion of patients with vulnerable plaque was $33.3 \%$ in the mild stenosis group, $62.5 \%$ in the moderate stenosis group and $87.5 \%$ in the severe stenosis group. The proportions of vulnerable plaque in the three groups with differing degrees of stenosis 
were therefore different, and the more severe the stenosis, the higher the proportion of vulnerable plaque that was identified. However, vulnerable plaques were also identified in patients with only mild stenosis, which is a finding consistent with that of previous studies (35-37). This also revealed the danger that, if the degree of vascular stenosis is taken into consideration as the sole determining factor of clinical surgical intervention, the risk assessment is impaired and will result in some patients not receiving effective treatment.

At present, in all the common non-invasive clinical imaging methods used to evaluate carotid artery plaque, carotid artery ultrasound is widely used in clinical practice as it is simple, convenient and non-invasive and is associated with low costs. However, due to the limitations of space, time and resolution, the accuracy of ultrasound in the evaluation of carotid plaque properties is reduced (38). Therefore, carotid ultrasound is limited in its ability to identify vulnerable plaque. According to the degree of stenosis suggested by vascular ultrasound, mild stenosis, moderate stenosis and severe stenosis were classified. There is contrast in the analysis of plaque properties between ultrasound and HR-MRI in different degrees of stenosis when evaluating the vulnerability of plaque, and the current study suggested that HR-MRI is more specific, as the higher the stenosis rate, the heavier the vulnerable plaque rate. Compared with ultrasound, HR-MRI is an ideal method for the continuous examination of the artery wall, since it has a better ability to distinguish tissue characteristics (39-41). The comparative study of MRI and histopathology has revealed that MRI can accurately differentiate between the plaque fiber cap, IPH, LRNC and calcification (42), since different components have different signal characteristics in multi-contrast sequence images. A large number of comparative studies on imaging and pathology have demonstrated that HR-MRI produces results that are highly consistent with those of histopathology in identifying and quantitatively analyzing carotid atherosclerotic plaque $(13,17,43)$, and its levels of sensitivity and specificity have reached $90-100 \%$ accuracy $(12,13)$. The calcification components in atherosclerosis exhibit a low signal on T1WI, PDWI and T2 images (44). Saam et al (13) identified that HR-MRI has both high sensitivity and specificity in identifying calcified components (86 and 76\%, respectively). In terms of the imaging of plaque with or without a fiber cap, on the 3D-TOF sequence images, the complete thick fiber cap was identified to be a continuous low signal close to the vascular lumen in the image, and the signal was uniform (45). However, on the T1WI, PDWI and T2 sequence images, the signal was high. On the 3D-TOF sequence, the broken fiber cap was revealed as an interrupted low signal, or no signal, close to the vascular lumen in the image, and the lumen was found to be irregular on each sequence image (45). A subsequent study revealed that an important feature of vulnerable plaque is IPH, which is crucial for the stable development of vulnerable plaque (46). Different periods of IPH have different signal characteristics, as revealed by multi-contrast MRI sequences (47). Fresh IPH, with a bleeding time of $<1$ week, is identified by a high signal on T1WI and TOF sequence images, whereas it appears as an isointense or low signal on T2WI and precision diagnostic imaging (PDI) sequence images (47). Recent IPH with a bleeding time $>1$ and $<6$ weeks is revealed on T1WI, T2W and TOF sequence images, the images and
PDI sequence images often exhibit a clear high signal and the old bleeding with bleeding time $>6$ weeks is revealed as a low signal on T1WI sequence images, T2WI sequence images and TOF sequence images (47). These results confirm that HR-MRI can distinguish between the different stages of bleeding in advanced atherosclerotic carotid atherosclerotic plaques with high sensitivity and specificity (48). The presence and size of the LRNC is considered to be an important indicator of atherosclerotic plaque vulnerability (49-51). In the present study, HR-MRI based on multi-contrast MRI acquisition parameters combined with MRI-PlaqueView ${ }^{\mathrm{TM}}$ software could correctly identify fiber cap, IPH, LRNC and calcification, and distinguish these features from each other. Additionally, it was possible to quantify each component in the plaque by utilizing the differences in the different colors, based on the multi-dimensional color display of the plaque components. By comparing the types and image characteristics of plaque in carotid ultrasound, HR-MRI was indicated to have the ability to identify vulnerable plaque, and it is a more intuitive method with several clear advantages compared with ultrasonography. HR-MRI can qualitatively and quantitatively evaluate differences in the different shapes and components of carotid stable and vulnerable plaque in vivo, which will help patients with carotid disease and ischemic stroke to achieve better results in terms of risk stratification and management guidance $(52,53)$.

However, there are several limitations associated with the method that has been implemented in the present clinical study. Firstly, after the examination, the patients did not undergo a follow up examination at a later stage. Therefore, it was not clear whether the prognosis is worse if the blood vessel stenosis is more severe, or whether the prognosis is worse when more vulnerable plaques are present. Also, there is the possibility that some asymptomatic neck plaques can undergo a further stroke. Secondly, the sample size of the present case study was small, and there may be a certain bias associated with some of the conclusions. Thirdly, the patients included in the current study did not undergo endometrial exfoliation treatment, so plaque pathology examination was not possible. Lack of a pathological gold standard as a validation tool is a limitation to the current study. However, HR-MRI has been demonstrated to be the most sensitive and specific diagnostic tool for in vivo morphological characterization of atheromatous carotid artery plaques, which is strongly in agreement with histological findings (54). In future studies, it should be possible to further analyze a comparison of the plaque HR-MRI performance and pathology. Taken together, the results of the present study have clearly shown, however, that HR-MRI may identify plaque properties and quantitatively analyze them, and that this method has unique advantages in terms of the hierarchical management of ischemic stroke.

\section{Acknowledgements}

Not applicable.

\section{Funding}

Funding was received from the Jilin Province Science and Technology Project (grant no. 20190303163SF). 


\section{Availability of data and materials}

The datasets used and/or analyzed during the current study are available from the corresponding author on reasonable request.

\section{Authors' contributions}

YS interpreted and analyzed the data. LX wrote the manuscript, analyzed the data and designed the study. YJ and MM performed statistical analysis and data analysis. XW collected the ultrasound and MRI data of the patients, and acquired and interpreted data. YX reviewed the article and designed the study. All authors read and approved the final manuscript.

\section{Ethics approval and consent to participate}

The current study was approved by the Ethics Committee of China-Japan Union hospital of Jilin University (approval no. 2020010812). Patients who participated in this research had complete clinical data. Signed informed consents were obtained from the patients and/or the guardians.

\section{Patient consent for publication}

Not applicable.

\section{Competing interests}

The authors declare that they have no competing interests.

\section{References}

1. GBD 2016 Stroke Collaborators: Global, regional, and national burden of stroke, 1990-2016: A systematic analysis for the Global Burden of Disease Study 2016. Lancet Neurol 18: 439-458, 2019.

2. Habibi-Koolaee M, Shahmoradi L, Niakan Kalhori SR, Ghannadan H and Younesi E: Prevalence of stroke risk factors and their distribution based on stroke subtypes in Gorgan: A retrospective hospital-based study-2015-2016. Neurol Res Int 2018: 2709654, 2018.

3. Mead GE, Murray H, Farrell A, O'Neill PA and McCollum CN: Pilot study of carotid surgery for acute stroke. Br J Surg 84: 990-992, 1997.

4. Polak JF, Pencina Mj, O'Leary DH and D'Agostino RB: Common carotid artery intima-media thickness progression as a predictor of stroke in multi-ethnic study of atherosclerosis. Stroke 42: 3017-3021, 2011.

5. Li GW, Zheng Gy, Li JG and Sun XD: Relationship between carotid atherosclerosis and cerebral infarction. Chin Med Sci J 25: 32-37, 2010.

6. Fisher M, Paganini-Hill A, Martin A, Cosgrove M, Toole JF, Barnett HJ and Norris J: Carotid plaque pathology: Thrombosis, ulceration, and stroke pathogenesis. Stroke 36: 253-257, 2005.

7. Seeger JM, Barratt E, Lawson GA and Klingman N: The relationship between carotid plaque composition, plaque morphology, and neurologic symptoms. J Surg Res 58: 330-336, 1995.

8. van Lammeren GW, Reichmann BL, Moll FL, Bots ML, de Kleijn DP, de Vries JP, Pasterkamp G and de Borst GJ: Atherosclerotic plaque vulnerability as an explanation for the increased risk of stroke in elderly undergoing carotid artery stenting. Stroke 42: 2550-2555, 2011.

9. Howard DP, van Lammeren GW, Rothwell PM, Redgrave JN, Moll FL, de Vries JP, de Kleijn DP, den Ruijter HM, de Borst GJ and Pasterkamp G: Symptomatic carotid atherosclerotic disease: Correlations between plaque composition and ipsilateral stroke risk. Stroke 46: 182-189, 2015.
10. Brinjikji W, Huston J III, Rabinstein AA, Kim GM, Lerman A and Lanzino G: Contemporary carotid imaging: From degree of stenosis to plaque vulnerability. J Neurosurg 124: 27-42, 2016.

11. Sadat U, Teng Z, Young VE, Graves MJ, Gaunt ME and Gillard JH: High-resolution magnetic resonance imaging-based biomechanical stress analysis of carotid atheroma: A comparison of single transient ischaemic attack, recurrent transient ischaemic attacks, non-disabling stroke and asymptomatic patient groups. Eur J Vasc Endovasc Surg 41: 83-90, 2011.

12. Kang X, Polissar NL, Han C, Lin E and Yuan C: Analysis of the measurement precision of arterial lumen and wall areas using high-resolution MRI. Magn Reson Med 44: 968-972, 2000.

13. Saam T, Ferguson MS, Yarnykh VL, Takaya N, Xu D, Polissar NL, Hatsukami TS and Yuan C: Quantitative evaluation of carotid plaque composition by in vivo MRI. Arterioscler Thromb Vasc Biol 25: 234-239, 2005.

14. Peng B and $\mathrm{Wu} \mathrm{B}$ : Guidelines for the diagnosis and treatment of acute ischemic stroke in China 2018. Chinese J Neurol 9: 666-682, 2018

15. Grant EG, Benson CB, Moneta GL, Alexandrov AV, Baker JD, Bluth EI, Carroll BA, Eliasziw M, Gocke J, Hertzberg BS, et al: Carotid artery stenosis: Grayscale and Doppler ultrasound diagnosis-society of radiologists in ultrasound consensus conference. Ultrasound Q 19: 190-198, 2003.

16. Xu D, Hippe DS, Underhill HR, Oikawa-Wakayama M, Dong L, Yamada K, Yuan C and Hatsukami TS: Prediction of high-risk plaque development and plaque progression with the carotid atherosclerosis score. JACC Cardiovasc Imaging 7: 366-373, 2014.

17. Cai JM, Hatsukami TS, Ferguson MS, Small R, Polissar NL and Yuan C: Classification of human carotid atherosclerotic lesions with in vivo multicontrast magnetic resonance imaging. Circulation 106: 1368-1373, 2002.

18. Androulakis E, Norrington K, Bakogiannis C, Lioudaki E, Siasos G and Tousoulis D: The impact of antiplatelet treatment on endothelial function. Curr Pharm Des 22: 4512-4518, 2016.

19. Chaturvedi S, Bruno A, Feasby T, Holloway R, Benavente O, Cohen SN, Cote R, Hess D, Saver J, Spence JD, et al: Carotid endarterectomy-an evidence-based review: Report of the therapeutics and technology assessment subcommittee of the American Academy of Neurology. Neurology 65: 794-801, 2005.

20. Fairhead JF and Rothwell PM: The need for urgency in identification and treatment of symptomatic carotid stenosis is already established. Cerebrovasc Dis 19: 355-358, 2005.

21. Topakian R, King A, Kwon SU, Schaafsma A, Shipley M and Markus HS; ACES Investigators: Ultrasonic plaque echolucency and emboli signals predict stroke in asymptomatic carotid stenosis. Neurology 77: 751-758, 2011.

22. Saba L, Biswas M, Suri HS, Viskovic K, Laird JR, Cuadrado-Godia E, Nicolaides A, Khanna NN, Viswanathan V and Suri JS: Ultrasound-based carotid stenosis measurement and risk stratification in diabetic cohort: A deep learning paradigm. Cardiovasc Diagn Ther 9: 439-461, 2019.

23. Basic J, Assadian A, Strassegger J, Senekowitsch C, Wickenhauser G, Koulas S, Waldhör T and Duschek N: Degree of contralateral carotid stenosis improves preoperative risk stratification of patients with asymptomatic ipsilateral carotid stenosis. J Vasc Surg 63: 82-88.e2, 2016.

24. van den Bouwhuijsen QJ, Bos D, Ikram MA, Hofman A, Krestin GP, Franco OH, van der Lugt A and Vernooij MW: Coexistence of calcification, intraplaque hemorrhage and lipid core within the asymptomatic atherosclerotic carotid plaque: The Rotterdam Study. Cerebrovasc Dis 39: 319-324, 2015.

25. Chen XY, Wong KS, Lam WW, Zhao HL and Ng HK: Middle cerebral artery atherosclerosis: Histological comparison between plaques associated with and not associated with infarct in a postmortem study. Cerebrovasc Dis 25: 74-80, 2008.

26. Ananyeva NM, Kouiavskaia DV, Shima M and Saenko EL: Intrinsic pathway of blood coagulation contributes to thrombogenicity of atherosclerotic plaque. Blood 99: 4475-4485, 2002.

27. Wong KS, Gao S, Chan YL, Hansberg T, Lam WW, Droste DW, Kay R and Ringelstein EB: Mechanisms of acute cerebral infarctions in patients with middle cerebral artery stenosis: A diffusion-weighted imaging and microemboli monitoring study. Ann Neurol 52: 74-81, 2002.

28. Bogousslavsky J, Barnett HJ, Fox AJ, Hachinski VC and Taylor W: Atherosclerotic disease of the middle cerebral artery. Stroke 17: 1112-1120, 1986. 
29. Lu M, Peng P, Cui Y, Qiao H, Li D, Cai J and Zhao X: Association of progression of carotid artery wall volume and recurrent transient ischemic attack or stroke: A magnetic resonance imaging study. Stroke 49: 614-620, 2018.

30. Kerwin WS: Carotid artery disease and stroke: Assessing risk with vessel wall MRI. ISRN Cardiol 2012: 180710, 2012.

31. Dong L, Underhill HR, Yu W, Ota H, Hatsukami TS, Gao TL, Zhang Z, Oikawa M, Zhao X and Yuan C: Geometric and compositional appearance of atheroma in an angiographically normal carotid artery in patients with atherosclerosis. AJNR Am J Neuroradiol 31: 311-316, 2010.

32. Yoshida K, Yang T, Yamamoto Y, Kurosaki Y, Funaki T, Kikuchi T, Ishii A, Kataoka $\mathrm{H}$ and Miyamoto S: Expansive carotid artery remodeling: Possible marker of vulnerable plaque. J Neurosurg: 1-6, 2019.

33. Saam T, Underhill HR, Chu B, Takaya N, Cai J, Polissar NL, Yuan C and Hatsukami TS: Prevalence of American Heart Association type VI carotid atherosclerotic lesions identified by magnetic resonance imaging for different levels of stenosis as measured by duplex ultrasound. J Am Coll Cardiol 51: 1014-1021, 2008.

34. Cheung HM, Moody AR, Singh N, Bitar R, Zhan J and Leung G: Late stage complicated atheroma in low-grade stenotic carotid disease: MR imaging depiction-prevalence and risk factors. Radiology 260: 841-847, 2011.

35. Xu L, Wang R, Liu H, Wang J, Liang W, Mang J and Xu Z: Comparison of the diagnostic performances of ultrasound, high-resolution magnetic resonance imaging, and positron emission tomography/computed tomography in a rabbit carotid vulnerable plaque atherosclerosis model. J Ultrasound Med: May 12, 2020 (Epub ahead of print)

36. Takai H, Uemura J, Yagita Y, Ogawa Y, Kinoshita K, Hirai S, Ishihara M, Hara K, Toi $H$, Matsubara S, Nishimura $H$ and Uno M: Plaque characteristics of patients with symptomatic mild carotid artery stenosis. J Stroke Cerebrovasc Dis 27: 1930-1936, 2018.

37. Kashiwazaki D, Shiraishi K, Yamamoto S, Kamo T, Uchino H, Saito H, Akioka N, Kuwayama N, Noguchi K and Kuroda S: Efficacy of carotid endarterectomy for mild $(<50 \%)$ symptomatic carotid stenosis with unstable plaque. World Neurosurg 121: e60-e69, 2019

38. Anzidei M, Napoli A, Zaccagna F, Di Paolo P, Saba L, Cavallo Marincola B, Zini C, Cartocci G, Di Mare L, Catalano C and Passariello R: Diagnostic accuracy of colour Doppler ultrasonography, CT angiography and blood-pool-enhanced MR angiography in assessing carotid stenosis: A comparative study with DSA in 170 patients. Radiol Med 117: 54-71, 2012.

39. Toussaint JF, LaMuraglia GM, Southern JF, Fuster V and Kantor HL: Magnetic resonance images lipid, fibrous, calcified, hemorrhagic, and thrombotic components of human atherosclerosis in vivo. Circulation 94: 932-938, 1996.

40. Yuan C, Hatsukami TS, Beach KW, Hayes CE, Nelson JA, Ferguson MS, Clowes AW and Strandness E Jr: In vivo MR evaluation of atherosclerosis in human carotid artery with use of phased-array coils. J Vasc Interv Radiol 7: 46-48, 1996.

41. Toussaint JF, Southern JF, Fuster V and Kantor HL: T2-weighted contrast for NMR characterization of human atherosclerosis. Arterioscler Thromb Vasc Biol 15: 1533-1542, 1995.
42. den Hartog AG, Bovens SM, Koning W, Hendrikse J, Luijten PR, Moll FL, Pasterkamp G and de Borst GJ: Current status of clinical magnetic resonance imaging for plaque characterisation in patients with carotid artery stenosis. Eur J Vasc Endovasc Surg 45: 7-21, 2013.

43. Hatsukami TS, Ross R, Polissar NL and Yuan C: Visualization of fibrous cap thickness and rupture in human atherosclerotic carotid plaque in vivo with high-resolution magnetic resonance imaging. Circulation 102: 959-964, 2000

44. Cappendijk VC, Cleutjens KB, Kessels AG, Heeneman S, Schurink GW, Welten RJ, Mess WH, Daemen MJ, van Engelshoven JM and Kooi ME: Assessment of human atherosclerotic carotid plaque components with multisequence MR imaging: Initial experience. Radiology 234: 487-492, 2005.

45. Mitsumori LM, Hatsukami TS, Ferguson MS, Kerwin WS, Cai J and Yuan C: In vivo accuracy of multisequence MR imaging for identifying unstable fibrous caps in advanced human carotid plaques. J Magn Reson Imaging 17: 410-420, 2003.

46. Kolodgie FD, Gold HK, Burke AP, Fowler DR, Kruth HS, Weber DK, Farb A, Guerrero LJ, Hayase M, Kutys R, et al: Intraplaque hemorrhage and progression of coronary atheroma. N Engl J Med 349: 2316-2325, 2003.

47. Yang L, Li D and Wei Y: The clinical application and technology progress of high resolution MRI in carotid intraplaque hemorrhage. Chinese J Magn Reson Imaging 6: 711-715, 2015.

48. Chu B, Kampschulte A, Ferguson MS, Kerwin WS, Yarnykh VL, O'Brien KD, Polissar NL, Hatsukami TS and Yuan C: Hemorrhage in the atherosclerotic carotid plaque: A high-resolution MRI study. Stroke 35: 1079-1084, 2004.

49. Gutstein DE and Fuster V: Pathophysiology and clinical significance of atherosclerotic plaque rupture. Cardiovasc Res 41: 323-333, 1999.

50. Naghavi M, Libby P, Falk E, Casscells SW, Litovsky S, Rumberger J, Badimon JJ, Stefanadis C, Moreno P, Pasterkamp G, et al: From vulnerable plaque to vulnerable patient: A call for new definitions and risk assessment strategies: Part I. Circulation 108: 1664-1672, 2003.

51. Falk E: Stable versus unstable atherosclerosis: Clinical aspects. Am Heart J 138: S421-S425, 1999.

52. Li ZY, Tang T, U-King-Im J, Graves M, Sutcliffe M and Gillard JH: Assessment of carotid plaque vulnerability using structural and geometrical determinants. Circ J 72: 1092-1099, 2008.

53. Xia Z, Yang H, Yuan X, Wang J, Zhang S, Zhang L, Qu Y, Chen J, Jiao L, Wang LX and Du Y: High-resolution magnetic resonance imaging of carotid atherosclerotic plaques-a correlation study with histopathology. Vasa 46: 283-290, 2017.

54. Chiocchi M, Chiaravalloti A, Morosetti D, Loreni G, Gandini R, Mancino S, Fabiano S and Simonetti G: Virtual histology-intravascular ultrasound as a diagnostic alternative for morphological characterization of carotid plaque: Comparison with histology and high-resolution magnetic resonance findings. J Cardiovasc Med (Hagerstown) 20: 335-342, 2019.

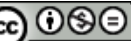

This work is licensed under a Creative Commons Attribution-NonCommercial-NoDerivatives 4.0 International (CC BY-NC-ND 4.0) License. 